Rakenteiden Mekaniikka (Journal of Structural Mechanics)

Vol. 51, No 2, 2018, pp. $49-61$

https://rakenteidenmekaniikka.journal.fi/index

https://doi.org/10.23998/rm.66836

(C) The author(s) 2018.

Open access under CC BY-SA 4.0 license.

\title{
Buckling length of a frame member
}

\author{
Teemu Tiainen ${ }^{1}$ and Markku Heinisuo
}

Summary. In the design of steel frames, the definition of buckling length of its members is a basic task. Computers can be used to calculate the eigenmodes and corresponding eigenvalues for the frame and using these the buckling length of the members can be defined by using the well-known Euler's equation. However, it is not always easy to say, which eigenmode should be used for the definition of the buckling length of a specific member. Conservatively, the lowest positive eigenvalue can be used for all members. In this paper, two methods to define the buckling length of a specific member are presented. The first one uses geometric stiffness matrix locally and the other one uses strain energy measures to identify members taking part in a buckling mode. The applicability of the methods is shown in several numerical examples. Both methods can be implemented into automated frame design, removing one big gap in the integrated design. This is essential when optimization of frames is considered.

Key words: effective length, frame analysis, elastic buckling

Received 6 November 201\%. Accepted 19 October 2018. Published online 8 December 2018.

\section{Introduction}

In the code-based structural design, the buckling length (also effective length) or load of a member is still an important design parameter. For certain structures, the design codes and standards give values for the length factors, as is the case with tubular trusses in EN 1993-1-1 for example, but in general the task is left to the designer.

Multiple methods have been proposed for finding the effective length of a frame member. Widely used simplified approach has been presented by Dumonteil [1]. In this contribution, the transcendental equation is solved approximately with simplified formulas. Multiple extensions for this work have been carried out by several other authors. For example, semi-rigid joints have been considered in [5]. Webber et al. [8] has proposed an extension to cover the effect of axial force in columns adjoining the considered member as well as the effect of axial force in other columns in the same floor. In the examples, it is shown that this approach gives very accurate values in comparison to results given by a finite element software.

Even if the presented simplified methods can be considered to be accurate enough to be applicable with design codes, they do not necessarily fit well in integrated design systems.

${ }^{1}$ Corresponding author. teemu.tiainen@tut.fi 
For example, in approach proposed by Webber et al., the user needs to identify other columns in the floor which is not always straightforward task in a complicated structure.

It should also be noted that according to standards, such as the EN 1993-1-1, the concept of buckling length is not needed if geometrically non-linear analysis is employed. However, application of non-linear models will result in greater computational effort needed for the analysis. In case of a single analysis, this is clearly not a problem with contemporary computational tools. However, when optimization is performed the analysis needs to be carried out multiple - even thousands of - times.

Therefore, in this contribution, two approaches for a programmable procedure to assess the effective length are presented. The methods cannot practically be used with hand calculation but need a finite element code that can be altered. However, the routines needed for the proposed methods are relatively easy to implement for an experienced user. The paper is organized as follows. The concept of buckling length and the methods are presented in the following sections and their performance is evaluated in three numerical examples.

\section{Linear stability analysis and buckling length}

Typical approach using linear stability theory for elastic buckling yields the well-known linear system of equations

$$
\mathbf{f}=\mathbf{K u}
$$

where $\mathbf{f}$ is the vector of nodal forces, $\mathbf{K}$ is the stiffness matrix, to be solved for nodal displacements $\mathbf{u}$. With the nodal displacements, respective internal forces in the elements can be calculated

$$
\mathbf{f}^{e}=\mathbf{k}^{e} \mathbf{u}^{e}+\mathbf{r}^{e}
$$

where $\mathbf{f}^{e}$ is the vector of internal forces in element $e, \mathbf{k}^{e}$ is the stiffness matrix of element $e, \mathbf{u}^{e}$ is the vector of displacements for the element $e$ and $\mathbf{r}^{e}$ is the vector of equivalent external nodal loads.

For the linear stability analysis, the axial forces in each element are picked to form a geometrical stiffness matrix $\mathbf{K}_{g}$ which is used for writing an eigenvalue problem

$$
\left(\mathbf{K}+\lambda \mathbf{K}_{g}\right) \mathbf{q}=\mathbf{0}
$$

where $\lambda$ is the eigenvalue and $\mathbf{q}$ is the respective eigenvector representing the buckling mode.

When assessing the buckling length of a single member with the finite element approach it should be recognized which eigenpair should be used. Let us assume it is pair with eigenvalue $\lambda_{j}$ for member $i$.

The critical axial force in flexural buckling by axis $y$ is defined by

$$
N_{c r, y}=\frac{\pi^{2} E I_{y}}{L_{c r, y}^{2}}
$$

where $E$ is the Young's modulus for the material, $I_{y}$ is the second moment of the section in the plane of buckling and $L_{c r, y}$ is the critical length or buckling length for buckling about $y$ axis. Nomenclature and axis definition follow those of EN 1993-1-1 (see Fig. 1). From this expression, the buckling length can be solved

$$
L_{c r, y}=\pi \sqrt{\frac{E I_{y}}{N_{c r, y}}}
$$



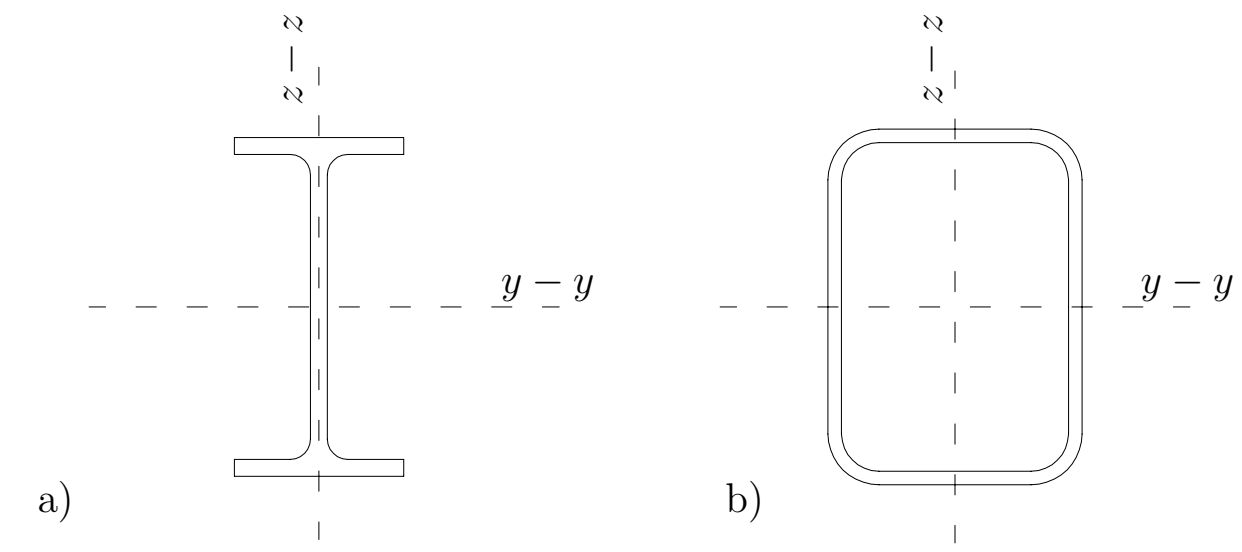

Figure 1: Local axes definition according to EN 1993-1-1 for a) I section b) rectangular hollow section.

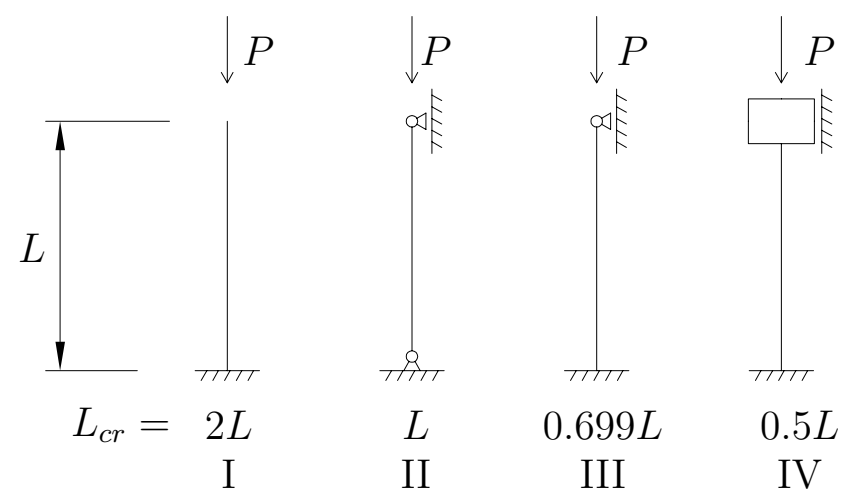

Figure 2: Euler buckling cases.

On the other hand, for member $i$, the buckling force can be expressed with eigenvalue as $N_{c r, y, i}=\lambda_{j}\left|N_{i}\right|$, thus

$$
L_{c r, y}=\pi \sqrt{\frac{E I_{y}}{\lambda_{j}\left|N_{i}\right|}}
$$

The buckling length can be best understood when it is compared to the member system length $L_{\text {sys }}{ }^{2}$. This can be assessed by formula

$$
L_{c r, y}=k L_{\text {sys }}
$$

where $k$ is a buckling length factor for given direction of buckling (also referred to as $\mathrm{K}$-factor in literature). In the well-known Euler cases the factor gets values shown in Tab. 2 but typical members in real frames or other structures rarely fit these support conditions.

\section{Proposed approach with local geometric stiffness}

To help the task of choosing the correct eigenpair, the first proposed idea is to include only the finite elements in the evaluated member in the eigenproblem

$$
\left(\mathbf{K}+\lambda \mathbf{K}_{g}^{i}\right) \mathbf{q}=\mathbf{0}
$$

${ }^{2}$ The system length is a concept used by EN 1993-1-1. The length means member length in the mechanical model. 
in which

$$
\mathbf{K}_{g}^{i}=\sum \mathbf{K}_{g}^{e}
$$

in which the sum is taken over elements belonging to member $i$.

This implies that instead of one eigenproblem, the design engineer should solve as many eigenproblems as there are compressed members in the structure. However, it is very straightforward that only the lowest positive eigenvalue from each analysis is used.

\section{Proposed approach with strain energy measure}

The second proposed approach is based on strain energy. In the well-known linear finite element framework, the element strain energy is calculated as

$$
E^{e}=\frac{1}{2} \mathbf{q}^{T} \mathbf{k}^{e} \mathbf{q}
$$

where $\mathbf{k}^{e}$ is the element stiffness matrix and $\mathbf{q}$ is the vector of displacements. Moreover, the member strain energy can be calculated as

$$
E^{m}=\frac{1}{2} \mathbf{q}^{T} \sum \mathbf{k}^{e} \mathbf{q}
$$

Respectively, for the whole structure, the total strain energy can be calculated when the global stiffness matrix $\mathbf{K}$ is used

$$
E=\frac{1}{2} \mathbf{q}^{T} \mathbf{K q}
$$

The ratio for each member in a deformed shape can be thus calculated as

$$
R_{m}=\frac{E^{m}}{E}
$$

This can be done separately for each member and each eigenmode with positive eigenvalue. The problem related to scaling of eigenvectors disappears when only the relation to total strain energy is considered.

To judge whether a single member is taking part in a buckling mode, it is assumed that the member will have a substantial share of the total strain energy. The share which can be considered substantial is, however, not easily judged. The initial proposal for the criterion is

$$
R_{m} \geq \frac{1}{n}
$$

where $n$ is number of members in the structure.

The rationale behind the proposal is purely empirical, based on manual trials on several rectangular 2D frames. However, more rigorous testing might be needed to find out a general criterion to suit other types of structures (frames with diagonal members et cetera).

\section{Numerical examples}

Three numerical examples are considered. The first two are extremely simple and academic with only two members but they show some very basic features about the methods rather nicely. The third is an example of a more realistic design situation where a tubular steel truss is connected to columns forming a building frame. 


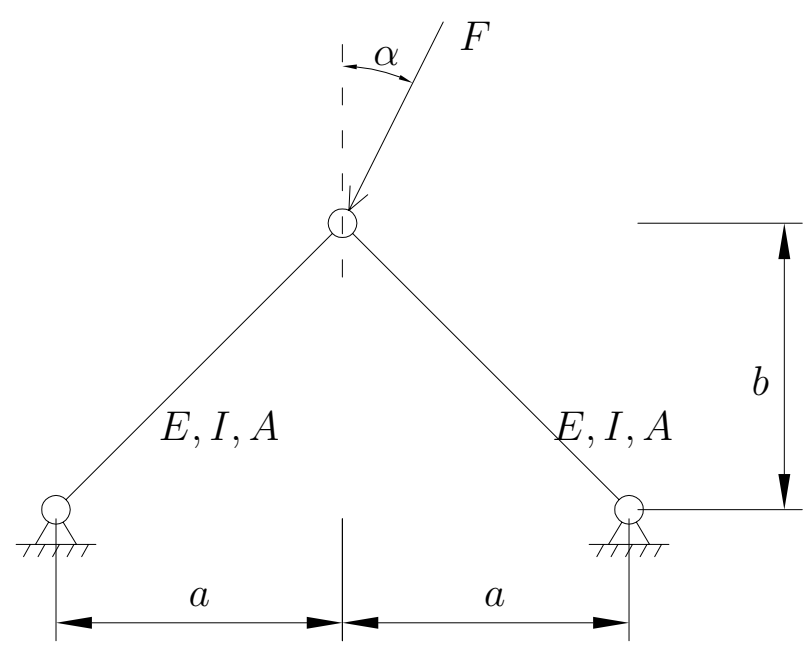

Figure 3: Two member truss.

\section{Two member truss}

Consider a two member truss in Fig. 3. Each member is modeled by using five EulerBernoulli beam elements with element stiffness matrix

$$
\mathbf{k}=\frac{E}{l}\left[\begin{array}{cccccc}
A & 0 & 0 & -A & 0 & 0 \\
& \frac{12 I}{l^{2}} & \frac{6 I}{l} & 0 & -\frac{12 I}{l^{2}} & \frac{6 I}{l} \\
& & 4 I & 0 & -\frac{6 I}{l} & 2 I \\
& & & A & 0 & 0 \\
\text { sym } & & & & \frac{12 I}{l^{2}} & -\frac{6 I}{l} \\
& & & & & 4 I
\end{array}\right]
$$

and element geometric stiffness matrix

$$
\mathbf{k}_{g}=\frac{1}{30 l}\left[\begin{array}{cccccc}
0 & 0 & 0 & 0 & 0 & 0 \\
& 36 & 3 l & 0 & -36 & 3 l \\
& & 4 l^{2} & 0 & -3 l & -l^{2} \\
& & & 0 & 0 & 0 \\
& & & & 36 & -3 l \\
\text { sym } & & & & & 4 l^{2}
\end{array}\right]
$$

The ratio of measures $a / b$ is set to 1 . The buckling length is calculated with ten values of angle $\alpha$. The results are seen in Tab. 1. The local approach gives exact value for buckling length factor with each value. The situation is illustrated for $\alpha=40^{\circ}$ in Fig. 4 where it can be seen that first three modes represent the buckling of left member and the fourth represents the mode where the member on the right hand side buckles. The respective shares of strain energies are shown in Tab. 2.

Clearly, both proposed methods give exact values for buckling length of both members. The use of lowest eigenmode will result error that grows when $\alpha$ approaches $45^{\circ}$. When $\alpha=45^{\circ}$, there is no compressive force in the member 2 and therefore buckling force or buckling length factor cannot be calculated.

\section{Two member frame}

Consider a two member frame in Fig. 5. Similarly to previous example, five EulerBernoulli elements are used in modeling each member. 
Table 1: Buckling length factors with local approach for the two-member truss.

\begin{tabular}{ccccc}
\hline & \multicolumn{2}{c}{ Local k $\mathbf{k}_{g}$} & \multicolumn{2}{c}{ Lowest eigenmode } \\
$\alpha\left[^{\circ}\right]$ & $k_{1}[-]$ & $k_{2}[-]$ & $k_{1}[-]$ & $k_{2}[-]$ \\
\hline 0 & 1.00 & 1.00 & 1.00 & 1.00 \\
5 & 1.00 & 1.00 & 1.00 & 1.09 \\
10 & 1.00 & 1.00 & 1.00 & 1.19 \\
15 & 1.00 & 1.00 & 1.00 & 1.32 \\
20 & 1.00 & 1.00 & 1.00 & 1.46 \\
25 & 1.00 & 1.00 & 1.00 & 1.66 \\
30 & 1.00 & 1.00 & 1.00 & 1.93 \\
35 & 1.00 & 1.00 & 1.00 & 2.38 \\
40 & 1.00 & 1.00 & 1.00 & 3.38 \\
45 & 1.00 & - & 1.00 & - \\
\hline
\end{tabular}

Table 2: Relative strain energy of the five lowest modes in the two-member truss example.

\begin{tabular}{cccccc}
\hline & \multicolumn{5}{c}{ Mode } \\
Member & 1 & 2 & 3 & 4 & 5 \\
\hline Left & 1.00 & 1.00 & 1.00 & 0.00 & 1.00 \\
Right & 0.00 & 0.00 & 0.00 & 1.00 & 0.00 \\
\hline
\end{tabular}

The corresponding results as in previous example are shown for this example in Tabs. 3 and 4 . In this example, the buckling of members is coupled with small $\alpha$ values. This implies error for the local approach. With the energy based approach the buckling of member with higher load is clearly correct but the buckling of the other member gets very low buckling length values. With $\alpha=25^{\circ}$, there is not even a mode within the first ten lowest ones which would give strain energy content of $50 \%$ (see Eq. 14) or more. However, in Tab. 5 it can be seen that in the third mode the strain energy content of the member is 49 \%. Thus, it seems that the rule specified in Eq. 14 is not applicable in this example.

\section{Truss frame}

Consider a truss frame in Fig. 6. The structure is constructed from cold formed square hollow sections with member profile dimensions shown in Tab. 6. The chosen profiles are a result of optimization [7] with fixed values of buckling length proposed by EN 1993-1-1 for the truss members and simply $0.9 \mathrm{~L}$ for the columns. Cross-sectional properties are calculated following EN 10219-2 [2]. In the mechanical model, the brace-to-chord and chord-to-column joints are hinged, chords and columns are continuous and modeled with beam elements without hinges.

In standard [3], the buckling length factor value for chords is 0.9 and for braces 0.9 (According to Finnish national annex for EN 1993-1-1, value 0.75 can be used). For braces, the model with ideal hinges gives length factor $k=1.0$ and the value suggested by the standard is lower. This is due to fact that in welded tubular trusses the joints are not necessarily ideally hinged but semi-rigid.

Therefore, some rotational stiffness for the joints is approximated. Joint fixity factor 
Table 3: Buckling length factors with local approach for the two-member frame.

\begin{tabular}{ccccccc}
\hline & \multicolumn{3}{c}{ Local $\mathbf{k}_{g}$} & \multicolumn{2}{c}{ Energy } & \multicolumn{2}{c}{ Lowest eigenmode } \\
$\alpha\left[^{\circ}\right]$ & $k_{1}[-]$ & $k_{2}[-]$ & $k_{1}[-]$ & $k_{2}[-]$ & $k_{1}[-]$ & $k_{2}[-]$ \\
\hline 0 & 0.84 & 0.84 & 1.00 & 0.70 & 1.00 & 1.00 \\
5 & 0.84 & 0.84 & 0.96 & 0.73 & 0.96 & 1.05 \\
10 & 0.84 & 0.84 & 0.93 & 0.76 & 0.93 & 1.11 \\
15 & 0.84 & 0.84 & 0.91 & 0.79 & 0.91 & 1.20 \\
20 & 0.84 & 0.84 & 0.89 & 0.41 & 0.89 & 1.31 \\
25 & 0.84 & 0.84 & 0.88 & - & 0.88 & 1.46 \\
30 & 0.84 & 0.84 & 0.87 & 0.41 & 0.87 & 1.68 \\
35 & 0.84 & 0.84 & 0.86 & 0.69 & 0.86 & 2.05 \\
40 & 0.84 & 0.84 & 0.85 & 0.72 & 0.85 & 2.90 \\
45 & 0.84 & - & 0.84 & - & 0.84 & - \\
\hline
\end{tabular}

Table 4: Relative strain energy of first five modes in the two-member frame, $\alpha=40^{\circ}$.

\begin{tabular}{cccccc}
\hline & \multicolumn{5}{c}{ Mode } \\
Member & 1 & 2 & 3 & 4 & 5 \\
\hline Left & 0.80 & 0.89 & 0.92 & 0.87 & 0.40 \\
Right & 0.20 & 0.11 & 0.08 & 0.13 & 0.60 \\
\hline
\end{tabular}

Table 5: Relative strain energy of first five modes in the two-member frame, $\alpha=25^{\circ}$.

\begin{tabular}{cccccc}
\hline & \multicolumn{5}{c}{ Mode } \\
Member & 1 & 2 & 3 & 4 & 5 \\
\hline Left & 0.74 & 0.66 & 0.51 & 0.73 & 0.55 \\
Right & 0.26 & 0.33 & 0.49 & 0.27 & 0.44 \\
\hline
\end{tabular}

Table 6: Truss-frame profile dimensions.

\begin{tabular}{ccc}
\hline Member & $b[\mathrm{~mm}]$ & $t[\mathrm{~mm}]$ \\
\hline Top Chord & 150 & 8 \\
Bottom Chord & 150 & 5 \\
Columns & 180 & 8 \\
Brace $(20 \& 33)$ & 100 & 4 \\
Brace $(21 \& 32)$ & 90 & 3 \\
Brace $(22 \& 31)$ & 90 & 3 \\
Brace $(23 \& 30)$ & 60 & 4 \\
Brace $(24 \& 29)$ & 60 & 4 \\
Brace $(25 \& 28)$ & 50 & 3 \\
Brace $(26 \& 27)$ & 50 & 3 \\
\hline
\end{tabular}




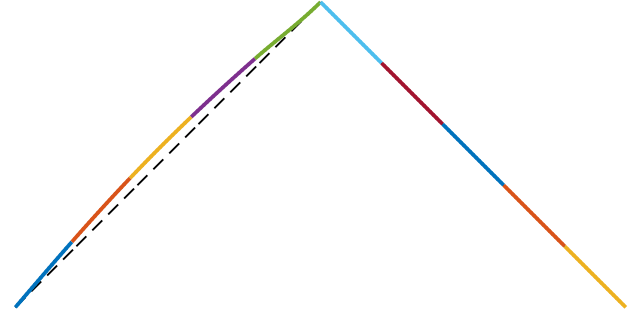

(a) Mode 1.

Critical load factor $\lambda_{\mathrm{cr}}=90.7732 \quad$ (LC=1.)

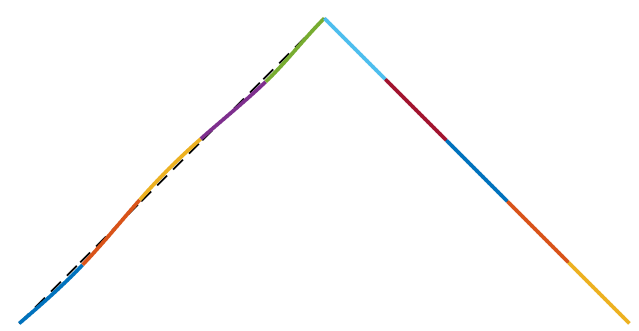

(c) Mode 3.

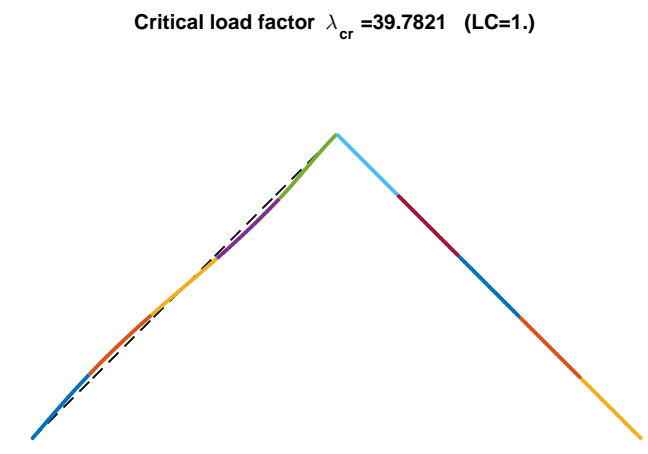

(b) Mode 2.

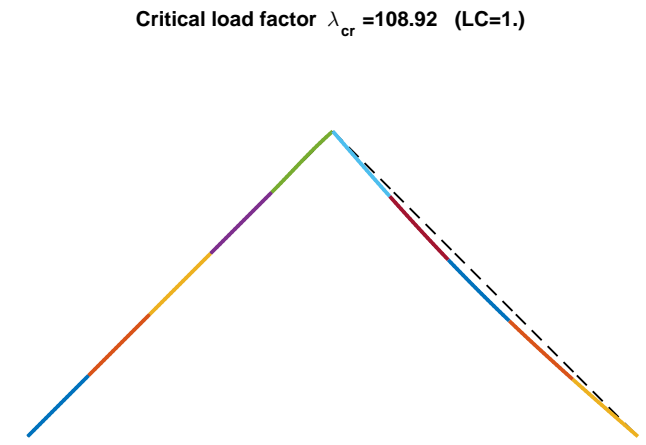

(d) Mode 4 .

Figure 4: Four lowest buckling modes for the two member truss. Colouring shows the used finite element division.

$\alpha[6]$ is defined as

$$
\alpha=\frac{1}{1+\frac{3 E I}{k L}}
$$

where $k$ is the rotational stiffness of the joint. Clearly, $\alpha=0$ means ideal hinge and $\alpha=1$ ideally rigid connection. The standard EN 1993-1-8 [4] specifies upper limit for ideally hinged joint to be

$$
k \leq \frac{E I}{2 L}
$$

this means

$$
\alpha=\frac{1}{7} \approx 0.143
$$

In the calculation, fixity factor value $\alpha=0.1$ is assumed for brace-to-chord joints. Every member is modeled again with five elements. The results for relative strain energy in ten lowest positive modes can be seen in Table 7. In this structure, the member that is buckling exhibits over $90 \%$ share of the total strain energy. Therefore, it is clear which member buckles in the first ten modes. This can be verified from Fig. 7 for the eight lowest modes.

The lowest four modes are connected to buckling of braces and the fifth one is a sway mode connected to column buckling. Upper chord buckling is seen in mode eight. The respective buckling length factors are seen in Table 8 .

It seems that the local approach can predict the buckling length of braces (members from 20 to 33) consistently. In the column buckling mode (Fig. 7 mode 5) both columns 


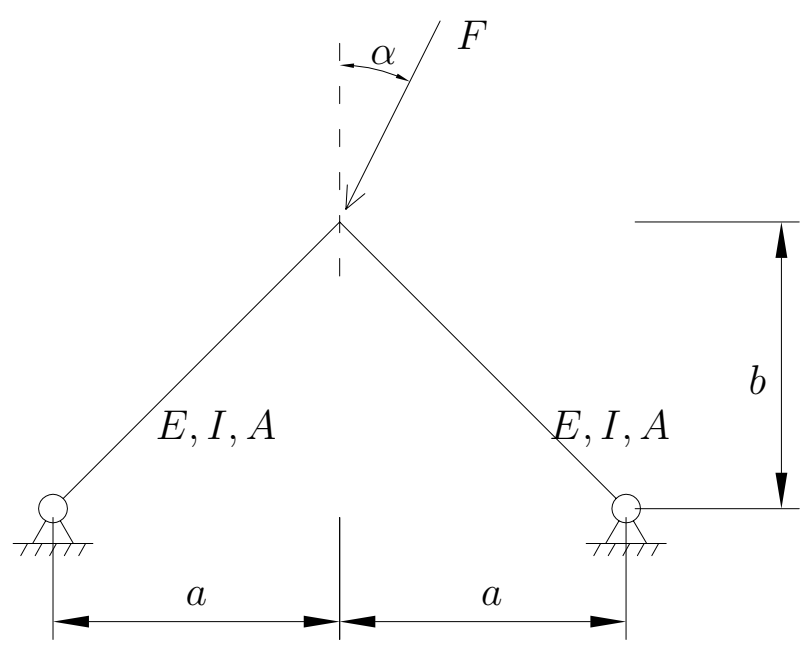

Figure 5: Two member frame.

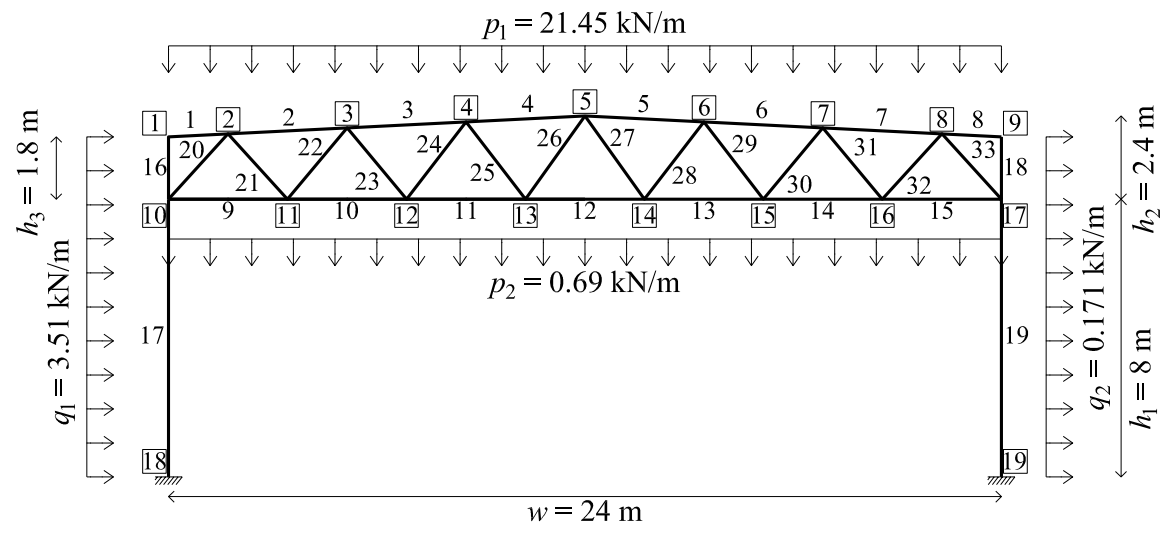

Figure 6: Truss frame example structure, loads and member numbering.

Table 7: Relative strain energy of members of the truss frame in ten lowest positive modes.

\begin{tabular}{ccccccccccc}
\hline & \multicolumn{1}{c}{ Mode } \\
Member & 1 & 2 & 3 & 4 & 5 & 6 & 7 & 8 & 9 & 10 \\
\hline Columns & 0.00 & 0.00 & 0.00 & 0.00 & 0.98 & 0.01 & 0.01 & 0.00 & 0.00 & 0.00 \\
Upper chord & 0.00 & 0.00 & 0.01 & 0.01 & 0.00 & 0.01 & 0.01 & 0.94 & 0.03 & 0.01 \\
Brace $(20 \& 33)$ & 0.00 & 0.00 & 0.00 & 0.00 & 0.02 & 0.98 & 0.98 & 0.00 & 0.00 & 0.00 \\
Brace $(21 \& 32)$ & 0.00 & 0.00 & 0.00 & 0.00 & 0.00 & 0.00 & 0.00 & 0.00 & 0.00 & 0.00 \\
Brace $(22 \& 31)$ & 0.00 & 0.00 & 0.99 & 0.99 & 0.00 & 0.00 & 0.00 & 0.01 & 0.00 & 0.00 \\
Brace $(23 \& 30)$ & 0.00 & 0.00 & 0.00 & 0.00 & 0.00 & 0.00 & 0.00 & 0.00 & 0.00 & 0.00 \\
Brace $(24 \& 29)$ & 1.00 & 1.00 & 0.00 & 0.00 & 0.00 & 0.00 & 0.00 & 0.05 & 0.97 & 0.99 \\
Brace $(25 \& 28)$ & 0.00 & 0.00 & 0.00 & 0.00 & 0.00 & 0.00 & 0.00 & 0.00 & 0.00 & 0.00 \\
Brace $(26 \& 27)$ & 0.00 & 0.00 & 0.00 & 0.00 & 0.00 & 0.00 & 0.00 & 0.00 & 0.00 & 0.00 \\
\hline
\end{tabular}


Table 8: Buckling length factors of compressed members obtained with the proposed methods and with the lowest eigenmode.

\begin{tabular}{cccc}
\hline Member & Local approach & Energy method & Lowest eigenmode \\
\hline 1 & 0.80 & 1.38 & 30.84 \\
2 & 0.67 & 1.18 & 1.97 \\
3 & 0.70 & 0.96 & 1.59 \\
4 & 0.70 & 0.91 & 1.52 \\
5 & 0.70 & 0.92 & 1.52 \\
6 & 0.70 & 0.97 & 1.61 \\
7 & 0.67 & 1.22 & 2.03 \\
16 & 0.95 & 24.65 & 31.38 \\
17 & 0.77 & 1.08 & 1.38 \\
18 & 0.95 & 27.62 & 35.17 \\
19 & 0.77 & 1.07 & 1.36 \\
20 & 0.80 & 0.80 & 1.13 \\
22 & 0.80 & 0.80 & 1.01 \\
24 & 0.80 & 0.80 & 0.83 \\
26 & 0.80 & 0.80 & 3.27 \\
27 & 0.80 & 0.80 & 1.70 \\
29 & 0.80 & 0.80 & 0.80 \\
31 & 0.80 & 0.80 & 0.99 \\
33 & 0.80 & 0.80 & 1.12 \\
\hline
\end{tabular}

buckle simultaneously and the roof sways horizontally. Thus geometrical stiffness matrix would have to be applied in both columns to capture the mode accurately. By doing this, eigenvalue 2.691 and buckling length factor 1.07 are obtained. These values are very close to the sway mode values.

With the energy method, members 16 and 18 have seemingly very high buckling length factor. However, if the respective buckling forces (Eq. 4) are calculated and the member resistance evaluated according to EN 1993-1-1, approximately $39 \%$ utilization ratio is obtained for members 16 and 18 whereas approximately $50 \%$ ratio is found in members 17 and 19. Thus the high buckling length factor for members 16 and 18 does not seem to have effect on the sizing of the column.

\section{Discussion and conclusions}

Both proposed methods are tested in three examples. The first two represent simplified and extreme structures with only two members. In the first one, the buckling modes are totally uncoupled. In the second one the coupling is very strong and there are some parameter values with which the methods fail to give correct buckling length values.

The local approach seems to work well in structures where eigenmodes are not coupled to other members' behaviour or coupling is moderate. If a sway buckling mode is expected, the global geometric stiffness matrix should be used or local matrix should be applied to all columns. However, to authors' knowledge, most of the typical structures found in buildings are braced (no sway modes) and connected with semirigid joints. This means that the applicability range of the method covers many typical structures. Moreover, the 
method is very straightforward to be implemented.

The approach based on energy measures can be considered very accurate but at this stage it is not clear how to formulate a criterion for choosing the correct eigenmode in a way that it would work with all frame structures. With the rather simple criterion proposed in the text, good results are obtained in the third design example but the second one reveals that the criterion is not general. Thus more research is needed for a more general criterion.

The computational effort is an important evaluation aspect of structural analysis methods in structural optimization where the objective and constraint functions may need to be evaluated thousands of times in a single optimization run. Of the two proposed methods, the energy based method seems more efficient since only one set of eigenpairs needs to be solved whereas in the local approach the eigenvalue solution needs to be repeated for each compressed member.

In the examples, prismatic members with Euler-Bernoulli beam assumptions are considered. However, both of the methods can theoretically handle also non-prismatic members and also distributed axial loading. Moreover, other types of assumptions of beam behaviour such as the Timoshenko beam theory and respective finite elements can be used with these methods.

In this contribution, only planar steel frames were considered. Thus, in further studies, extension to three dimensional structures including flexural buckling out-of-plane as well as torsional buckling behaviour should be considered.

\section{Acknowledgement}

The financial support of Finnish Cultural Foundation, Pirkanmaa Regional fund is gratefully acknowledged.

\section{References}

[1] Pierre Dumonteil. Simple equations for effective length factors. Eng J AISC, 29(3): 111-115, 1992.

[2] EN 10219-2. Cold formed welded structural hollow sections of non-alloy and fine grain steels. Part 2: Tolerances, dimensions and sectional properties. CEN, 2006.

[3] EN 1993-1-1. EN-1993-1-1. Eurocode 3: Design of steel structures. Part 1-1: General rules and rules for buildings. CEN, 2006.

[4] EN 1993-1-8. EN-1993-1-8. Eurocode 3: Design of steel structures. Part 1-8: Design of joints. CEN, 2006.

[5] Georgios E. Mageirou and Charis J. Gantes. Buckling strength of multi-story sway, non-sway and partially-sway frames with semi-rigid connections. Journal of Constructional Steel Research, 62(9):893 - 905, 2006. ISSN 0143-974X. doi:https://doi.org/10.1016/j.jcsr.2005.11.019.

[6] G. R. Monforton and Tien Hsing Wu. Matrix analysis of semi-rigid connected frames. Journal of the Structural Division, 89:13-24, 1963. 
[7] R. Van Mellaert, K. Mela, T. Tiainen, M. Heinisuo, G. Lombaert, and M. Schevenels. Mixed-integer linear programming reformulation approach for global discrete sizing optimization of trussed steel portal frames. In Kai-Uwe Bletzinger, Sierk Fiebig, Kurt Maute, Axel Schumacher, and Thomas Vietor, editors, WCSMO-12, Germany, 2017. doi:https://doi.org/10.1007/978-3-319-67988-4_56.

[8] A. Webber, J.J. Orr, P. Shepherd, and K. Crothers. The effective length of columns in multi-storey frames. Engineering Structures, 102:132-143, 2015. doi:https://doi.org/10.1016/j.engstruct.2015.07.039.

Teemu Tiainen, Markku Heinisuo

Tampere university of technology

PO box 60133101 Tampere, Finland

teemu.tiainen@tut.fi, markku.heinisuo@tut.fi 


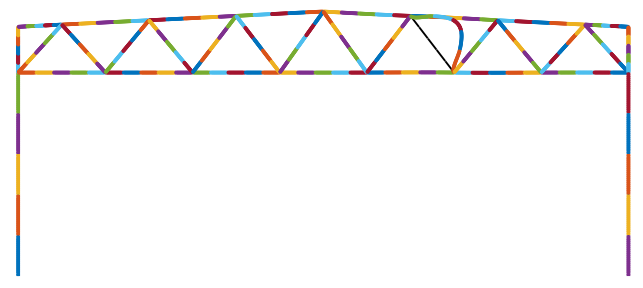

(a) Mode 1 .

Critical load factor $\lambda_{\text {cr }}=2.5386 \quad(L C=1$.)

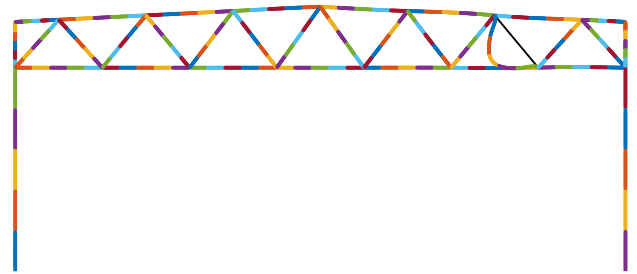

(c) Mode 3.

Critical load factor $\lambda_{\mathrm{cr}}=2.6819 \quad(\mathrm{LC}=1$.

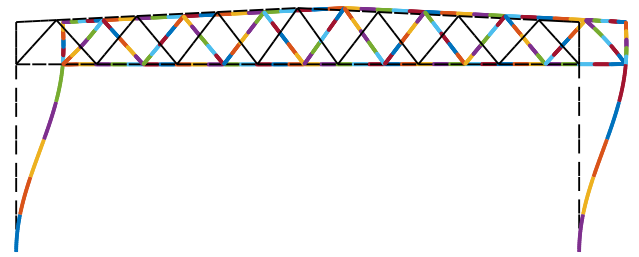

(e) Mode 5 .

Critical load factor $\lambda_{\mathrm{cr}}=3.3212 \quad$ (LC=1.)

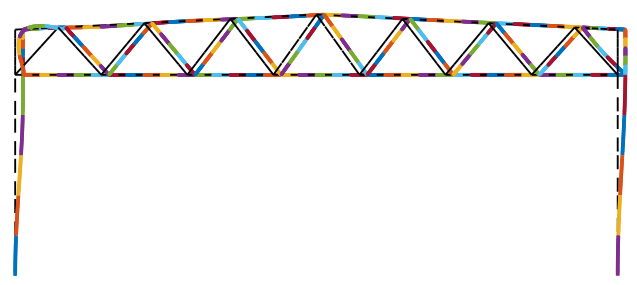

(g) Mode 7.

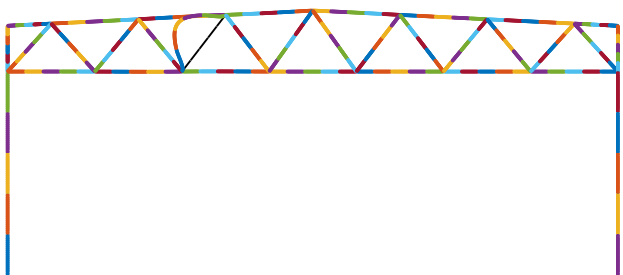

(b) Mode 2 .

Critical load factor $\lambda_{\mathrm{cr}}=2.6411 \quad(\mathrm{LC}=1$.)

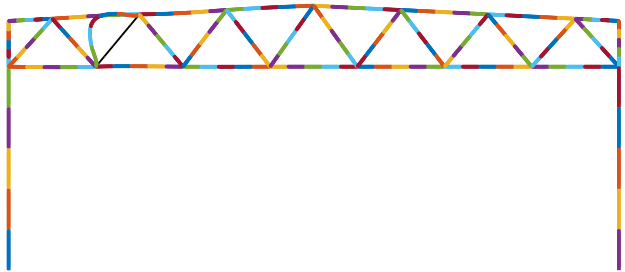

(d) Mode 4.

Critical load factor $\lambda_{\mathrm{cr}}=3.2292 \quad(\mathrm{LC}=1$.)

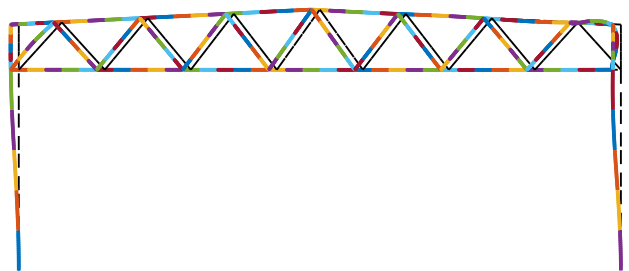

(f) Mode 6 .

Critical load factor $\lambda_{\mathrm{cr}}=4.5598 \quad(\mathrm{LC}=1$.

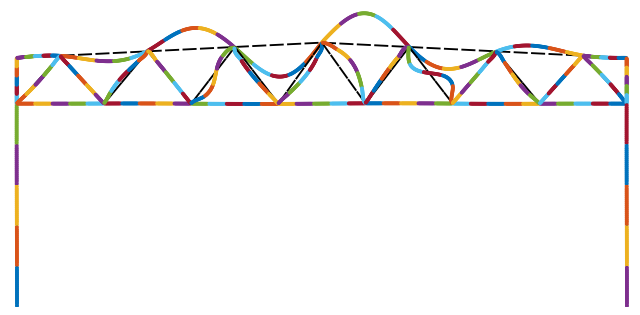

(h) Mode 8 .

Figure 7: First eight buckling modes for truss frame. 